and long-term effects of deutetrabenazine treatment on weight and metabolic parameters in individuals treated for TD.

Methods. Two 12-week, randomized placebo-controlled trials (RCTs) of deutetrabenazine for patients with TD evaluated either fixed dosing (AIM-TD; 12, 24, or $36 \mathrm{mg}$ ) or dose titration (ARMTD; max dose, $48 \mathrm{mg} /$ day). Patients completing ARM-TD or AIM-TD were included in an open-label extension (OLE) study, in which all patients underwent response-driven titration of deutetrabenazine from $12 \mathrm{mg} /$ day up to a maximum total dose of $48 \mathrm{mg}$ /day. Weight, body mass index (BMI), serum glucose, serum total cholesterol, and serum triglycerides were evaluated at baseline and during treatment in the RCTs and in the OLE.

Results. In the RCTs, 282 and 133 patients received deutetrabenazine or placebo. At baseline, $77 \%$ of patients used DRAs. At Week 12, no meaningful changes in weight were observed, with mean (standard error) weight changes of 0.9-1.2 (0.3-0.5) and 0.2 $(0.3) \mathrm{kg}$ in the deutetrabenazine and placebo groups, respectively, and mean BMI changes of 0.3-0.5 (0.1-0.2) and $0.1(0.1) \mathrm{kg} / \mathrm{m}^{2}$. 337 patients were included in the analysis of the OLE study. No meaningful changes were observed in weight (mean change: 0.4 $[0.4] \mathrm{kg}$ at Week 54, $-0.5[0.6] \mathrm{kg}$ at Week 106, and $-1.1[0.6] \mathrm{kg}$ at Week 145) or BMI (mean change: $0.1[0.2] \mathrm{kg} / \mathrm{m}^{2}$ at Week $54,-0.2$ $[0.2] \mathrm{kg} / \mathrm{m}^{2}$ at Week 106, and $-0.3[0.2] \mathrm{kg} / \mathrm{m}^{2}$ at Week 145). Across the studies, no meaningful changes were observed in triglyceride, cholesterol, or glucose levels.

Conclusion. Deutetrabenazine does not affect common metabolic parameters in patients with TD, even during long-term exposure.

Funding. Teva Pharmaceutical Industries Ltd., Petach Tikva, Israel

\section{Weight Gain and Treatment Interruptions with Second- Generation Oral Antipsychotics: Analysis of Patients with Schizophrenia or Bipolar I Disorder}

Michael J. Doane, $\mathrm{PhD}^{1}$, Leona Bessonova, $\mathrm{PhD}^{1}$, Kathleen Mortimer, $\mathrm{ScD}, \mathrm{MPH}^{2}$, Harry Cheng, MA, MPH${ }^{2}$, Gregory Donadio, $\mathrm{MA}^{2}$, Thomas Brecht, $\mathrm{MS}^{2}$, Amy K. O'Sullivan, $\mathrm{PhD}^{1}$, Hannah Cummings, $\mathrm{PhD}^{1}$, David McDonnell, $\mathrm{MD}^{3}$ and Jonathan M. Meyer, $\mathrm{MD}^{4}$

${ }^{1}$ Alkermes, Inc., Waltham, MA, USA, ${ }^{2} \mathrm{OM} 1$, Inc., Boston, MA, USA, ${ }^{3}$ Alkermes Pharma Ireland Ltd., Dublin, Ireland, and ${ }^{4}$ University of California, San Diego School of Medicine, Department of Psychiatry, La Jolla, CA, USA

Presenting Author: Michael J. Doane

\section{Abstract}

Among patients with schizophrenia (SZ) and bipolar I disorder (BD-I) treated with second-generation antipsychotics (SGAs),

clinically-significant weight gain (CSWG) and treatment interruptions (TIs) are challenges that may result in morbidity/mortality. CSWG and TIs were assessed among patients who initiated oral SGAs of moderate-to-high weight gain risk (no exposure to index SGAs/first-generation antipsychotics for $=12$ months) using medical records/claims (OM1 Data Cloud; January 2013February 2020). Outcomes included CSWG (=7\% increase in baseline weight) and TIs (switches [to SGAs of low weight gain risk/long-acting injectables] or discontinuations [no SGAs for $>30$ days]). Descriptive analyses included proportions of patients with CSWG and TIs, and median time to these outcomes.

Approximately three-quarters of patients were overweight/obese at baseline (SZ: N=8,174; BD-I: N=9,142). Within 3 months of SGA initiation, $12 \%$ of all patients experienced CSWG. For patients on treatment with index SGAs for $>6$ months (SZ: 29\%; BD-I: 27\%), $28 \%$ (SZ) and 30\% (BD-I) experienced CSWG during follow-up. Median time to CSWG was 14 weeks. CSWG results were numerically similar among patients with SZ and BD-I.

Over $96 \%$ of patients had TIs during follow-up (median time of 12 [SZ] and 13 [BD-I] weeks). Among patients with CSWG and subsequent TIs and weight measurements, $74 \%$ did not return to baseline weight after interrupting treatment; the remainder returned to baseline weight with median times of 38 (SZ) and 39 (BD-I) weeks. Results suggest that most patients with CSWG do not return to baseline weight after stopping treatment with oral SGAs of moderate-to-high weight gain risk.

Funding. Alkermes, Inc.

\title{
Othello Syndrome: Delusional Disorder - Jealous Type $\neq$ Violence
}

Elizabeth Soyeon Ahn, MD and

Jacqueline A. Hobbs, MD, PhD

University of Florida College of Medicine, Department of Psychiatry, Gainesville, FL, USA

Presenting Author: Elizabeth Soyeon Ahn

\section{Abstract}

Background. Othello syndrome, also known as morbid jealousy, pathological jealousy, and conjugal paranoia, is a rare delusional disorder related to partner's infidelity. There are no large scale or comprehensive studies on delusional jealousy, and only few case reports and cases series leave delusional disorder jealous type (DDJT) largely unknown. Herein, we report a case of DDJT, its possible etiology and describe its characteristics, comorbidities, and interventions.

Case Description. A 65-year-old married, retired, and disabled Caucasian male with a history of closed traumatic brain injury and chronic pain presented for outpatient care accompanied by his wife with a chief complaint of paranoid delusions. The patient was a car racer when he sustained over $25 \%$ total body surface area burns after his motor vehicle crashed at the speed of almost 
$160 \mathrm{mph}$. The patient was in a coma for more than nine weeks, coded three times, and was resuscitated each time. Per imaging, the patient suffered subarachnoid hemorrhage to the right outer parietal and left front parietal lobes. The patient developed chronic pain from the extensive burns and has been on opioids for many years until he gradually tapered himself off about six years ago. For the last couple of years, the patient has experienced cognitive decline associated with disorientation and memory deficit. The patient has been perseverative on his wife's trip two years ago during which the patient believed that she had an affair with one of their mutual acquaintances. The patient denied visual or auditory hallucinations and continued to express his love and affection toward his wife. Although his wife has continually provided reassurance, and multiple family members confirmed her faithfulness, the patient had minimal insight into his delusion and expressed disappointment in loss of sexual intimacy between him and his wife. The patient has been prescribed duloxetine $60 \mathrm{mg}$ and trazodone $150 \mathrm{mg}$, and his mood, anxiety, and sleep have been stable. The patient has also started taking pimozide $1 \mathrm{mg}$ nightly since beginning of this year with good response.

Discussion. The DSM estimates the prevalence of DDJT to be less than $1 \%$. At least a third of cases show neurological basis involving frontal lobe dysfunction associated with strokes, Parkinson's disease, brain trauma and tumors, neurodegenerative disorder, encephalitis, multiple sclerosis, and even normal pressure hydrocephalus. Association with alcohol, amphetamine, cocaine, and dopamine therapy (pergolide, ropinirole, levodopa, amantadine, and pramipexole) were reported. Finally, DDJT is known to be a risk factor of violent crimes including homicide. Treatment with pimozide shows the strongest evidence, and most patients show improvement with any antipsychotic medication along with CBT. Continued research and further clinical trials are warranted for DDJT considering patients' positive response to interventions, and because DDJT can become a dangerous condition in forensic situations.

\section{Economic Burden of Dementia Related Psychosis Among Medicare Beneficiaries: A State- Transition Markov Analysis of Total Annual Direct Costs}

\author{
Kinpritma Sangha, $\mathrm{PhD}, \mathrm{MPH}^{1}$, \\ Nazia Rashid, PharmD, MS' , Victor Abler, $\mathrm{MD}^{2}$ and \\ Krithika Rajagopalan, PhD, MS $^{1}$
}

\footnotetext{
${ }^{1}$ An-L-It-Iks, Inc., Framingham, MA, USA, and ${ }^{2}$ ACADIA Pharmaceuticals Inc., San Diego, CA, USA
}

Presenting Author: Kinpritma Sangha

\section{Abstract}

Study Objective. Dementia related psychosis (DRP), characterized by debilitating symptoms such as hallucinations and delusions, is estimated to affect $2.4 \mathrm{M}$ people with dementia in the US. Patients with DRP may have twice the rate of dementia progression compared to patients with no DRP. Given that dementia disproportionally impacts the elderly, a comprehensive cost of-illness analysis may add to the current understanding of the overall economic burden of DRP prevalence. The objective of this study was to estimate the cost of DRP from a Centers for Medicare and Medicaid Services (CMS) perspective.

Methods. A five state-transition Markov analysis, adapted from Green et al, was conducted to assess the annual direct DRP cost burden to CMS. Patients entering the model were allowed to transition between three at-home health-states (mild dementia plus psychosis, moderate dementia plus psychosis, severe dementia plus psychosis), one long-term care/nursing home (LTC/NH) stay, or death (absorbent health state) at any given time. Since the model accommodates tunnel health-states based on dementia, psychosis and functioning severity levels, patients stayed in the same health-state or transitioned to a more severe health-state or progressed to death (i.e., absorbent health-state) between each 30-day cycle. Prevalence, disease-severity, and state-transition probability estimates were derived from literature while direct costs of DRP were derived from a Medicare claims analysis. Costs were discounted at 3\%. Model robustness was tested to check if results were sensitive to changes in inputs and assumptions.

Results. Of the estimated 61.5M Medicare beneficiaries, about $6.87 \mathrm{M}$ may suffer from dementia. In the base-case scenario, an estimated total of $2.2 \mathrm{M}$ prevalent DRP patients enter the model based on dementia severity into one of the 4 non-absorbent health-states: three at-home (10\% mild, $60 \%$ moderate, and $10 \%$ severe) and one LTC-NH (10\%). Total Medicare annual direct DRP costs are estimated to be approximately $\$ 119.98 \mathrm{~B}$ (\$113.96B-\$125.96B) and about \$54K PPPY (Per-Patient-PerYear) costs (2019 USD). NH costs and patient volume at higher severity levels are significant cost drivers. Sensitivity analysis results show that the model is sensitive to disease severity and disease progression.

Conclusions. These results suggest that DRP imposes a significant direct cost burden despite its low prevalence. In this analysis, per-patient per year (PPPY) cost of DRP prevalence was estimated to be slightly higher than incident PPPY DRP costs. These differences may be attributed to the number of patients at higher severity levels and the time spent in a severe health state as well as cost of LTC/NH stays. Especially given the ageing population in the US, DRP could become an increasing public health concern. There is a significant need for education and awareness about DRP cost burden.

Funding. Acadia Pharmaceuticals Inc. 